\title{
Hepatic Encephalopathy With Corticospinal Tract Involvement Demonstrated by Diffusion Tensor Tractography
}

\author{
Hyun Bang, MD, Hye Yeon Lee, MD, Bo-Ram Kim, MD, In-Sik Lee, MD, \\ Heeyoune Jung, MD, Seong-Eun Koh, MD, Jongmin Lee, MD
}

Department of Rehabilitation Medicine, Konkuk University School of Medicine and Konkuk University Medical Center, Seoul, Korea

\begin{abstract}
A 50-year-old man with liver cirrhosis and esophageal varix for 3 years was diagnosed with hematemesis and treated for a bleeding varix. However, bleeding recurred 11 days later, and he developed drowsiness with left hemiparesis. His left upper and lower extremity muscle strengths based on the manual muscle test at the onset were grade $2 / 5$ and $1 / 5$, respectively. The Babinski sign was positive. His serum ammonia level was elevated to $129.9 \mu \mathrm{g} / \mathrm{dL}$ (normal, 20-80 $\mu \mathrm{g} / \mathrm{dL}$ ). Magnetic resonance imaging revealed restriction on diffusion and T2hyperintensities with decreased apparent diffusion coefficient values in the bilateral frontoparietooccipital cortex. The effect was more severe in the right hemisphere and right parietooccipital cortices, which were compatible with hepatic encephalopathy. Although the patient's mental status recovered, significant left-sided weakness and sensory deficit persisted even after 6 months. Diffusion tensor tractography (DTT) performed 3 months post-onset showed decreased volume of the right corticospinal tract. We reported a patient with hepatic encephalopathy involving the corticospinal tract by DTT.
\end{abstract}

Keywords Hepatic encephalopathy, Diffusion tensor imaging, Rehabilitation

\section{INTRODUCTION}

Hepatic encephalopathy (HE), a major complication of liver cirrhosis, requires careful evaluation of clinical

Received March 12, 2014; Accepted July 15, 2014

Corresponding author: Jongmin Lee

Department of Rehabilitation Medicine, Konkuk University Medical Center, 120 Neungdong-ro, Gwangjin-gu, Seoul 143-729, Korea

Tel: +82-2-1588-1533, Fax: +82-2-2030-5379, E-mail: leej@kuh.ac.kr

(c) This is an open-access article distributed under the terms of the Creative Commons Attribution Non-Commercial License (http://creativecommons. org/licenses/by-nc/3.0) which permits unrestricted noncommercial use, distribution, and reproduction in any medium, provided the original work is properly cited.

Copyright (c) 2015 by Korean Academy of Rehabilitation Medicine symptoms and underlying neurological disorders [1]. Previous studies have described neuropsychiatric characteristics including disturbed consciousness and cognitive impairments, such as memory decline and concentration deficits in HE patients [2]. Some patients with HE have focal neurologic signs, such as hemiparesis, abnormal extra-ocular movements, sensory deficits, and apraxia $[3,4]$.

Although the clinical diagnosis of HE requires careful consideration, imaging is becoming a more important tool in detecting microstructural brain abnormalities in HE patients. Magnetic resonance imaging (MRI) including diffusion-weighted imaging (DWI) and diffusion 
tensor tractography (DTT) studies have improved our understanding of basic neuroanatomical and pathophysiological alterations in patients with HE. DWI allows the assessment of intracellular and extracellular water contents in the brain. DTT provides information on water mobility and tissue microstructure and organization by quantitative DTT metrics, i.e., fractional anisotropy (FA) and mean diffusivity [5]. Previous studies using DTT in HE patients focused on the functional connectivity as an important mechanism $[6,7]$. Studies on HE by DTT that involve the corticospinal tract (CST) have not been previously reported. We reported the sudden onset of focal neurologic signs in an HE patient that persisted for 6 months, as demonstrated by DTT.

\section{CASE REPORT}

A 50-year-old man with a 3-year history of liver cirrhosis and esophageal varix visited the emergency care center of our medical institution. The patient had hematemesis and melena and underwent endoscopic variceal ligation. The patient suffered from variceal bleeding after 11 days and developed drowsiness with left-sided hemipare- sis. Manual muscle test based on the Medical Research Council (MRC) scale was performed at the onset, demonstrating grades of $2 / 5$ and $1 / 5$ for left upper and left lower extremities, respectively. Moreover, the Babinski sign was bilaterally positive. The patient had an elevated serum ammonia level $(129.9 \mu \mathrm{g} / \mathrm{dL})$, normal plasma glucose level, and normal renal function. Arterial blood gas analysis revealed normal findings. The patient was afebrile, and his complete blood count was within normal limits. Moreover, computed tomography (CT) angiogram and brain perfusion CT scans at the onset showed no evidence of vascular abnormality (Fig. 1A). However, diffusion-weighted MRI at the onset revealed suspicious hyperintensity and a decreased apparent diffusion coefficient (ADC) along bilateral frontoparietooccipital cortices (Fig. 1B). MRI scans performed 3 days after the initial symptoms revealed a marked increase in the extent of the DWI and T2-hyperintensities with decreased ADC in the bilateral frontoparietooccipital cortex; the degree of intensity was higher in the right hemisphere and right parietooccipital cortex (Fig. 1C). Electroencephalography (EEG) demonstrated low amplitude and slow activity. However, no epileptiform discharge was
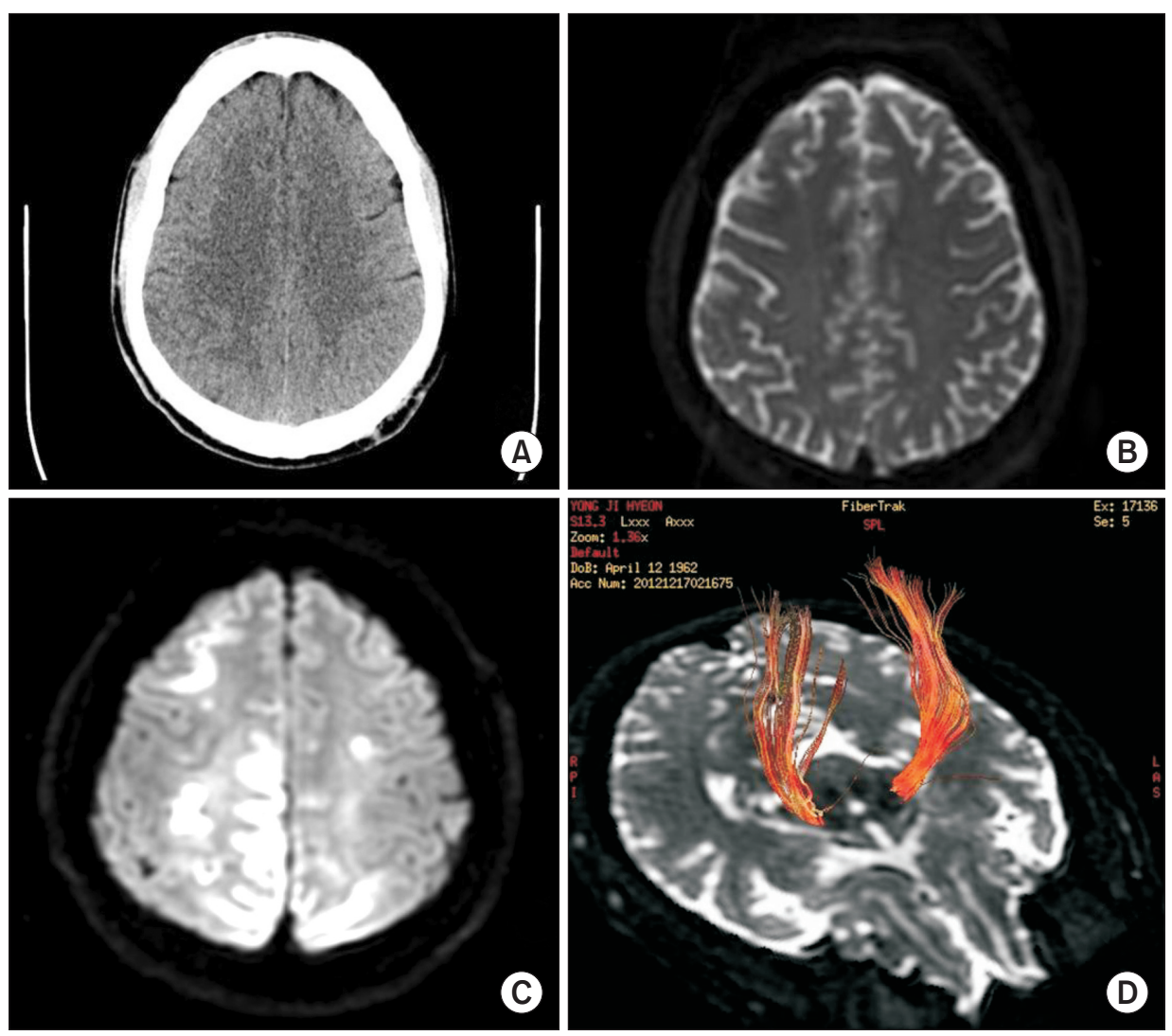

Fig. 1. (A) Absence of focal lesions in the brain parenchyma and cerebrospinal fluid spaces and other vascular abnormalities at the onset, on brain computed tomography (axial view). (B) Diffusionweighted imaging (DWI) at the onset showed suspicious hyperintensity and a decreased apparent diffusion coefficient (ADC) along the bilateral frontoparietoocipital cortices. (C) Brain magnetic resonance imaging scans 3 days after the onset showed increased intensity and a decreased ADC in the bilateral frontoparietooccipital (right>left) and right parietooccital cortex with subcortical vasogenic edema on the DWI. (D) Brain diffusion tensor tractography 2 months post-onset showed a relatively smaller volume of the right corticospinal tract. 
observed. The EEG findings were compatible with severe diffuse cerebral dysfunction. The patient's mental status recovered after stabilization of variceal bleeding and HE. The patient's Mini-Mental Status Examination score was 27 . Six weeks later, the patient was transferred to the department of rehabilitation medicine for further evaluation and treatment for the left-sided hemiparesis. The left-sided weakness and sensory deficit persisted for 2 months post-onset. According to the MRC, the muscle strength grades of left shoulder flexors, elbow flexors, and elbow extensors were $2 / 5,3 / 5$, and $3 / 5$, respectively, and those of left wrist flexors, extensors, and finger flexors were $4 / 5$. The muscle strength grades of the left hip flexors, knee flexors, and knee extensors were $1 / 5,2 / 5$, and $2 / 5$, respectively, and those of the left ankle dorsiflexors and plantarflexors were $1 / 5$. Two months after the onset of hemiparesis, the patient underwent DTT for an evaluation of the CST. A 3.0 Tesla MRI system (Signa HDxt; GE Medical Systems, Milwaukee, WI, USA) equipped with a standard 8-channel phase array head coil was used for obtaining a brain MRI scan and DTT measurements. For each of the 15 non-collinear and non-coplanar diffusionsensitizing gradients, approximately 70 contiguous slices parallel to the anterior commissure-posterior commissure line were aquired. Imaging parameters were as follows: matrix, $120 \times 120$; field of view, $240 \times 240 \mathrm{~mm}^{2}$; TE $=84$ $\mathrm{ms} ; \mathrm{TR}=16,000 \mathrm{~ms} ; \mathrm{b}=800 \mathrm{~mm}^{2} / \mathrm{s}$; and a slice thickness of $2 \mathrm{~mm}$. Conventional T2 fluid-attenuated inversion recovery images were obtained in addition to the diffusion tensor images. The data were transferred in digital imaging and communications in medicine format. All DTT images were corrected for eddy current-induced image distortions using the FSL software (The FMRIB Analysis Group, Oxford, UK; http://www.fmrib.ox.ac.uk/ fsl). DTT analyses were performed by an experienced rehabilitation medicine physician using the DTI studio (Johns Hopkins Medical Institute, Baltimore, MD, USA; http://cmrm.med.jhmi.edu). For reconstructing the CST, the first region of interest (ROI) was drawn free-hand in the posterior limb of the internal capsule through which the motor fibers descend; the second ROI was drawn in the basis pontis of lower pons using the 'AND' operation. The CST volume was calculated based on the number of the voxels in the reconstructed CST. The number of CST fibers, mean FA, and mean ADC values were calculated for the total tract. The MRI scan of the patient showed atrophy with hyperintensity of the bilateral frontoparietooccipital cortex, of higher intensity in the right parietooccipital cortices with subcortical area. Moreover, the DTT showed decreased volume of the right CST (Fig. 1D). Compared to the right CST, the left CST showed both decreased number of fibers and FA and an increased ADC value. The number of CST fibers was 2,612/1,060 (right side/left side). The FA and ADC values were $0.6456 / 0.6206$ and $0.6251 / 0.6124$ (right side/left side), respectively. After one month of intensive rehabilitation, the patient showed improvement in motor function; the muscle strength of all the left lower extremity muscles was $3 / 5$ except for the ankle dorsiflexors. The patient was able to walk using a quad cane and a left ankle-foot orthosis at the time of discharge. Three months post-discharge, which was 6 months post-onset, he was readmitted for intensive rehabilitation. Manual muscle test 6 months post-onset revealed left upper extremity and left lower extremity muscle strength grades of $4 / 5$ and $3 / 5$, respectively, except for the ankle dorsiflexor and plantarflexor strengths that were $1 / 5$. The patient was able to walk with a single cane and an ankle-foot orthosis. His daily living performance improved to the mildly dependent level with an improved Modified Barthel Index score of 79 at discharge, as compared to the initial score of 45 .

\section{DISCUSSION}

An HE diagnosis is routinely based on clinical patient observation; clinicians have to rule out other causes of altered mental status, such as hypoglycemia, mass lesions including subdural hematoma, cerebral abscess, primary central nervous system tumors, and metastatic tumors, seizures, and postictal states, migraine-mimicking stroke, encephalopathies, and other toxic-metabolite conditions [8]. Our patient had elevated serum levels of ammonia $(129.9 \mu \mathrm{g} / \mathrm{dL})$ but exhibited normal hepatic enzyme levels. Hypoglycemia was ruled out, since the glucose level was within normal limits. The patient had no signs of infection, and EEG findings were not indicative of epilepsy after the onset of left-sided hemiparesis. Moreover, CT angiogram and brain perfusion CT scans did not reveal any vascular abnormality. However, diffusion-weighted MRI scans showed an increased intensity and a decreased diffusion coefficient. The T2-weighted MRI scans revealed an increased intensity in the bilateral frontopa- 
rietooccipital cortex; the degree of intensity was higher in the right hemisphere and right parietooccipital cortex. These findings indicated the presence of hyperammonemic HE [9]. The pathogenesis explains the T2-high signal lesions that predominantly involve the hemispheric CST; the increased diffusivity in the hemispheric white matter in HE was brain edema [10].

The patient's mental status recovered, but there was a persistent left-sided weakness and sensory deficit even after 6 months. The patient was transferred to the department of rehabilitation medicine of our medical institution, and underwent DTT that revealed CST involvement in HE. Recently, according to the reports on DTT and HE, altered brain functional connectivity is an important mechanism in HE. Kale et al. [5] reported that minimal HE patients exhibit impaired microstructural integrity. Recently, some investigators demonstrated abnormal brain networks in patients with HE, such as the defaultmode network, dorsal attention network, visual network, and auditory network by functional MRI and DTT [6,7]. However, there are no studies regarding HE with CST involvement demonstrated by DTT. The study subject had persistent left-sided weakness and sensory deficit after the resolution of liver dysfunction. Irreversible focal neurologic signs in HE are rare, and to the best of our knowledge, focal neurologic signs due to CST involvement demonstrated by DTT have not been reported.

In conclusion, the study showed the possibility of CST damage despite the absence of major cerebrovascular abnormalities in patients with HE. Therefore, clinicians should also consider DTT as a diagnostic tool to evaluate the microstructural abnormalities in the brain, especially the CST in HE patients.

\section{CONFLICT OF INTEREST}

No potential conflict of interest relevant to this article was reported.

\section{REFERENCES}

1. Cordoba J. New assessment of hepatic encephalopathy. J Hepatol 2011;54:1030-40.

2. Mechtcheriakov S, Graziadei IW, Kugener A, Wiedemann J, Galbavy C, Hinterhuber H, et al. Multidimensional assessment of neuro-psychiatric symptoms in patients with low-grade hepatic encephalopathy: a clinical rating scale. World J Gastroenterol 2005; 11:5893-8.

3. Atchison JW, Pellegrino M, Herbers P, Tipton B, Matkovic V. Hepatic encephalopathy mimicking stroke: a case report. Am J Phys Med Rehabil 1992;71:114-8.

4. Yamamoto Y, Nishiyama Y, Katsura K, Yamazaki M, Katayama Y. Hepatic encephalopathy with reversible focal neurologic signs resembling acute stroke: case report. J Stroke Cerebrovasc Dis 2011;20:377-80.

5. Kale RA, Gupta RK, Saraswat VA, Hasan KM, Trivedi R, Mishra AM, et al. Demonstration of interstitial cerebral edema with diffusion tensor MR imaging in type C hepatic encephalopathy. Hepatology 2006;43:698706.

6. Chen HJ, Jiao Y, Zhu XQ, Zhang HY, Liu JC, Wen S, et al. Brain dysfunction primarily related to previous overt hepatic encephalopathy compared with minimal hepatic encephalopathy: resting-state functional MR imaging demonstration. Radiology 2013;266:26170.

7. Zhang LJ, Zheng G, Zhang L, Zhong J, Wu S, Qi R, et al. Altered brain functional connectivity in patients with cirrhosis and minimal hepatic encephalopathy: a functional MR imaging study. Radiology 2012;265:52836.

8. Prakash R, Mullen KD. Mechanisms, diagnosis and management of hepatic encephalopathy. Nat Rev Gastroenterol Hepatol 2010;7:515-25.

9. Bathla G, Hegde AN. MRI and CT appearances in metabolic encephalopathies due to systemic diseases in adults. Clin Radiol 2013;68:545-54.

10. Lizardi-Cervera J, Almeda P, Guevara L, Uribe M. Hepatic encephalopathy: a review. Ann Hepatol 2003; 2:122-30. 